\title{
TRADIZIONE E INNOVAZIONE NELLA PITTURA VENEZIANA DEL TRECENTO: PAOLO E ATTORNO A PAOLO
}

\author{
FRANCESCA D'ARCAIS
}

UDC 75 Paolo Veneziano 75(450) (091),13”

Original Scientific Paper

Manuscript received: 10. 01. 1996.

Revised manuscript accepted: 01. 04. 1996.

F. d'Arcais

Facoltà di Lettere e filosofia Università di Verona

Italia

Il linguaggio pittorico veneziano è anche nella prima metà del XIV secolo fortemente legato alla tradizione bizantina. Le novità compaiono con Maestro Paolo da Venezia, che traduce il linguaggio bizantino in un gotico sempre più moderno e raffinato. Ma la novità del suo stile non è subito recepita e accolta, nemmeno nelle grandi commissioni ufficiali, come la decorazione musiva del Battistero di San Marco, dovuta al Doge Andrea Dandolo.

Venezia è, come è ben noto, una sorta di provincia "bizantina", già fin dalle sue prime manifestazioni pittoriche. I più antichi mosaici di Torcello e di San Marco, che il Demus² data ancora entro il XI secolo, mostrano un linguaggio legatissimo a quello bizantino aulico; e nel percorso della decorazione musiva della basilica marciana tale legame non si interrompe mai, anche se le espressioni figurative si arricchiscono di stilemi e tipologie riconducibili, sempre secondo l' analisi del Demus, alla presenza di artisti locali: si coglie infatti in alcuni più briosi complessi, una varietà di invenzioni compositive e già una vivacità cromatica, che sembrano costituire una peculiarità linguistica tipicamente veneziana. Negli ultimi mosaici del XII secolo, quali gli episodi dell' arcone della Passione, e in quelli databili al primissimo Duecento, in particolare le "Storie del rinvenimento del corpo di San Marco" si accentuano ulteriormente quegli elementi che sembrano essere caratterizzanti appunto il linguaggio pittorico veneziano, e sono la vivacità narrativa, che sottolinea in maniera fortemente icastica i volti, o propone episodi di cronaca contemporanea, e la violenza degli accostamenti cromatici, scelti ancora per una resa più drammatica degli episodi (è il caso delle scene della Passione) o, per arricchire di particolari felicemente cronachistici, la narrazione.

Nei mosaici dell' atrio marciano, databili, sempre secondo il Demus tra il 1220 e il 1275, sono ancora questi gli elementi caratterizzanti le diverse composizioni, che arricchiscono dunque la eredità orientale, in una singolare ed efficacissima traduzione in un felicissimo "volgare" veneziano.

Nei decenni a cavallo tra la fine del XIII e gli inizi del XIV secolo il legame con l'arte bizantina non cessa di essere fortissimo, e Venezia, almeno nel campo pittorico, è e continua ad essere una "provincia" bizantina, anche se il rapporto non è più con il mondo aulico della capitale, ma piuttosto con i grandi centri della provincia, in particolare la Macedonia e la Serbia, territori del resto con i quali Venezia aveva stretti contatti economico-commerciali.

Gli affreschi bellissimi, provenienti dalla chiesa di San Zan Degolà, e che potrebbero datarsi ${ }^{3}$ alla fine del Duecento, o al

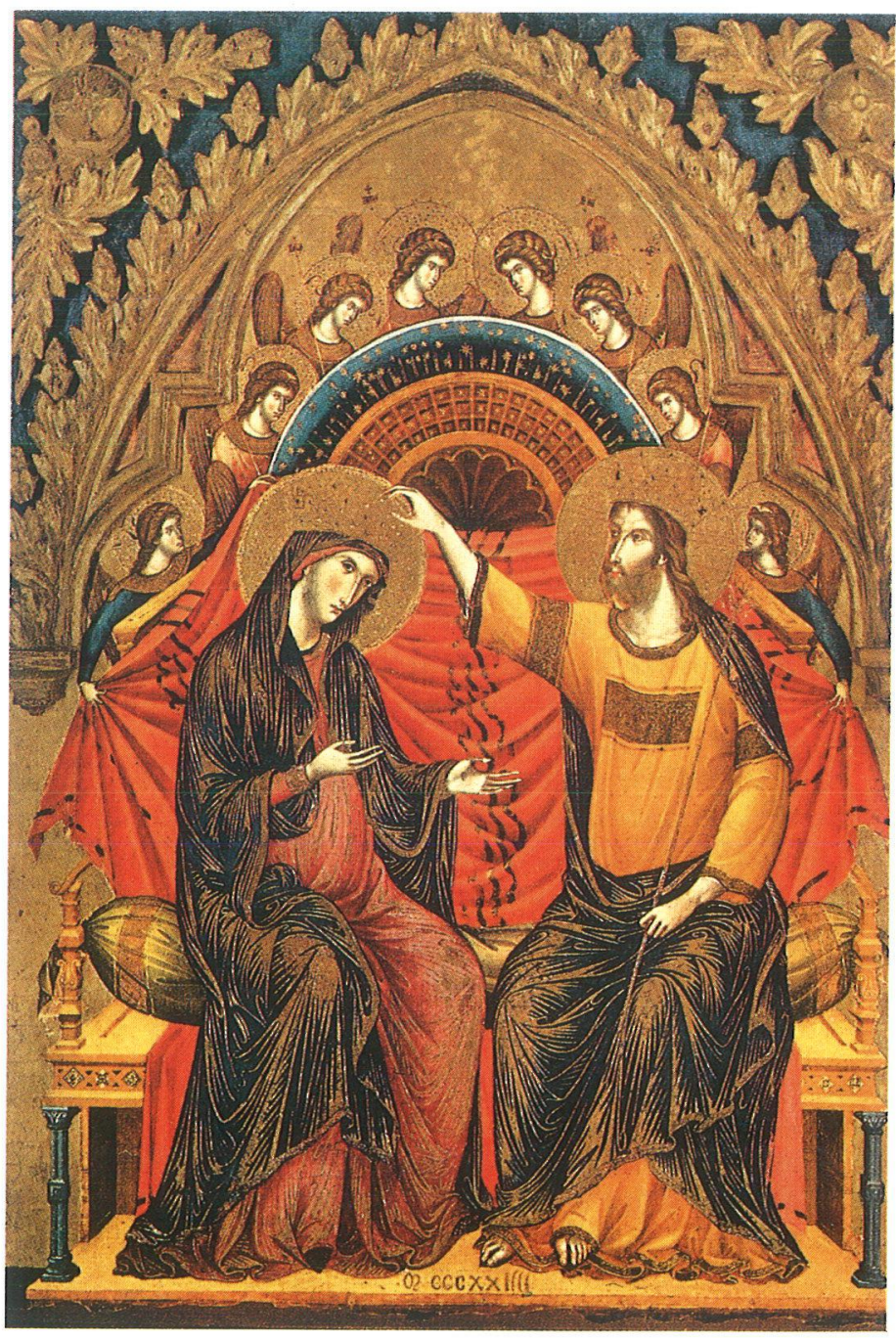

Fig. 1. Maestro Veneziano, "Incoronazione della Vergine"; Washington, Galleria Nazionale 


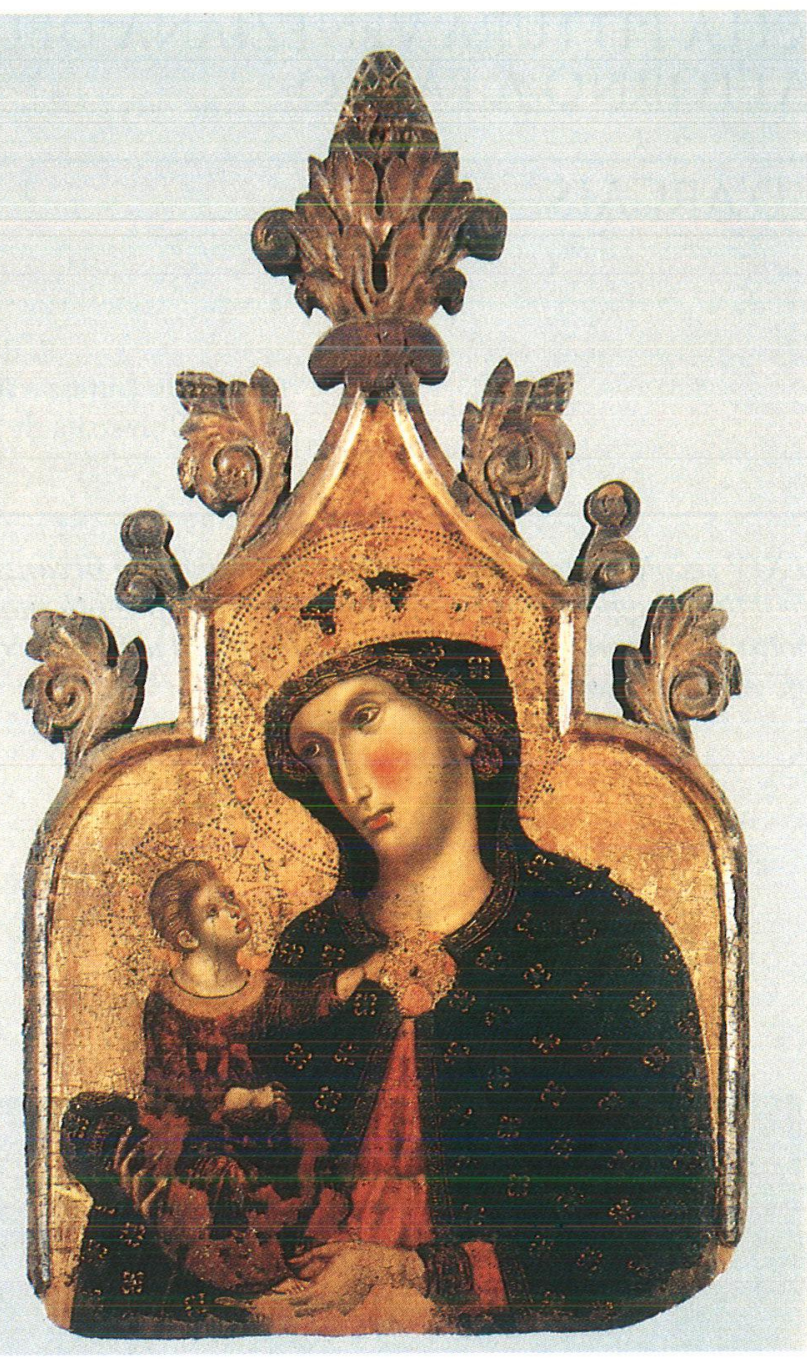

Fig. 2. Maestro dell'Incoronazione, Madonna e Bimbo; Padova, Museo Civico

massimo ai primissimi del Trecento, caratterizzati da una certa spazialità e da una volumetria di ricordo paleocristiano, mostrano ad esempio chiari rapporti con i modernissimi spaziosi e volumetrici affreschi della chiesa di Sopoćani, databili attorno al 1260. E anche in opere veneziane più tarde, l' accentuato linearismo dei contorni capace di ritagliare le figure sembra richiamare il linguaggio degli affreschi tardoduecenteschi della chiesa di San Clemente in Ocrida (1295).

Il giottismo che caratterizza la pittura degli altri centri italiani, almeno a partire dal secondo decennio del secolo, sembra non interessare Venezia, se non in maniera del tutto sporadica ad esempio nelle tavolette con "Storie della Vergine" del Museo Civico di Pesaro ${ }^{4}$, forse del terzo decennio, e nel Paliotto con "Storie del Beato Leone Bembo" di Dignano".

Ed è perciò che la pittura di Venezia, estranea di fatto alla rivoluzione "giottesca" che interessa tutti i centri artistici dell'area padana, appare attardata su posizioni isolate e tradizionali.

Le numerose opere veneziane, infatti, che si possono datare a cavallo tra il XIII e il XIV secolo, accuratamente elencate dal Garrison ${ }^{6}$ mostrano un linguaggio strettamente legato a quello orientale. Etale legame non si interrompe affatto anche nei dipinti ormai chiaramente trecenteschi ${ }^{7}$ : in questi piuttosto, il linguaggio si arricchisce di elementi tipici di Venezia, cioè la fioritura bellissima delle stoffe preziose, e di colori vivacissimi, che traducono in un felicissimo volgare veneziano la parlata bizantina.

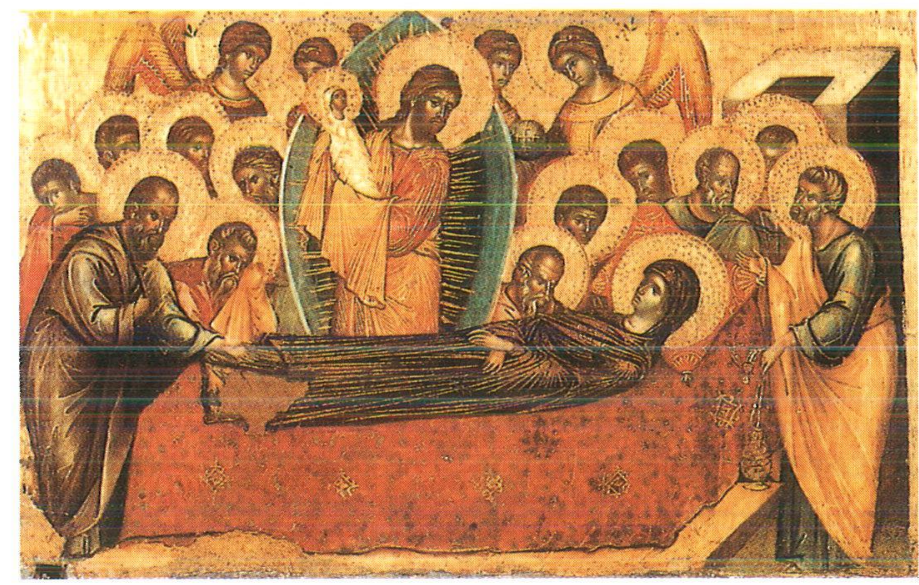

Fig. 3. Maestro Veneziano del primo Trecento, Dormizione della Vergine; Venezia, Chiesa San Pantalon

In questa chiave va letta la bellissima "Incoronazione" (fig. 1) della Galleria Nazionale di Washington, datata 1324, per la quale autorevoli critici hanno proposto il nome di Paolo Veneziano. Si tratta invece certamente di un grande pittore veneziano, cui il Lazareff, ${ }^{8}$ seguito dal Muraro e da altri, ha dato il nome di "Maestro dell' Incoronazione".

Se l'iconografia della "Incoronazione della Vergine" è occidentale, l'impianto delle figure è bizantino, nell'allungarsi dei corpi, che appare quasi senza peso, nelle tipologie dei volti e delle mani, che panni rigonfi ricoprono, ma non vestono. Ma il pittore è certo un veneziano nella straordinaria ricchezza del colori accostati in maniera raffinatissima e nello splendore degli ori. Alla "Incoronazione" sono state avvicinate ${ }^{9}$ la "Madonna in trono" del Museo Puskin di Mosca e la tavoletta frammentaria con una "Madonna col Bambino" del Museo Civico di Padova ${ }^{10}$ (fig. 2): in quest' ultimo dipinto tuttavia il grosso gioiello-fermaglio sul petto e la corona sul capo della Vergine, oltre ad evidenziare elementi gotici e non più bizantini, porterebbero a spostarne la datazione forse nel quarto decennio.

Un altro dipinto emblematico è il Polittico della chiesa di San Pantalon ${ }^{11}$, attribuito anche a Paolo: in questo la tradizione bizantina, seguita minutamente nelle "storiette", si arricchisce tuttavia di colori e di stoffe vivacissimi, già da leggersi in chiave gotica, e la Madonna propone al Figlio un papavero rosso, anch'esso elemento di squisita grazia gotica (figg. 3-4).

Ancora in chiave orientale va letta la parte centrale del trittico di Trieste ${ }^{12}$, ove le bellissime storiette presentano analoghi splendori di colore, così da tradurre ancora una volta la tradizione orientale in una felicissima e vivacissima narrazione, che è, come si è detto sopra, elemento tipico del linguaggio lagunare.

Anche nel campo della pittura di maggiori dimensioni nei primi decenni del XIV secolo è strettissimo il legame con la tradizione bizantina, e dal punto di vista iconografico e da quello stilistico: e si pensi alle due scene a fresco - unico resto di una più vasta decorazione - raffiguranti la "Crocifissione" ela "Deposizione di Cristo nel sepolcro", nella cappella Orlandini della chiesa dei SS. Apostoli a Venezia.

In questo contesto, fortemente tradizionale, ma anche già profondamente connotato di elementi locali, nasce e si sviluppa la personalità di Maestro Paolo ${ }^{13}$, che è certo la più nuova e la più alta di tutto il primo Trecento veneziano, ma che non costituisce l'unico parametro, come si vorrebbe, per giudicare la pittura veneziana.

La sua pittura acquisisce, e certo non potrebbe non farlo, il vasto patrimonio, specie iconografico, bizantino, ma lo traduce, con sempre maggiore coerenza, in un moderno lingu- 


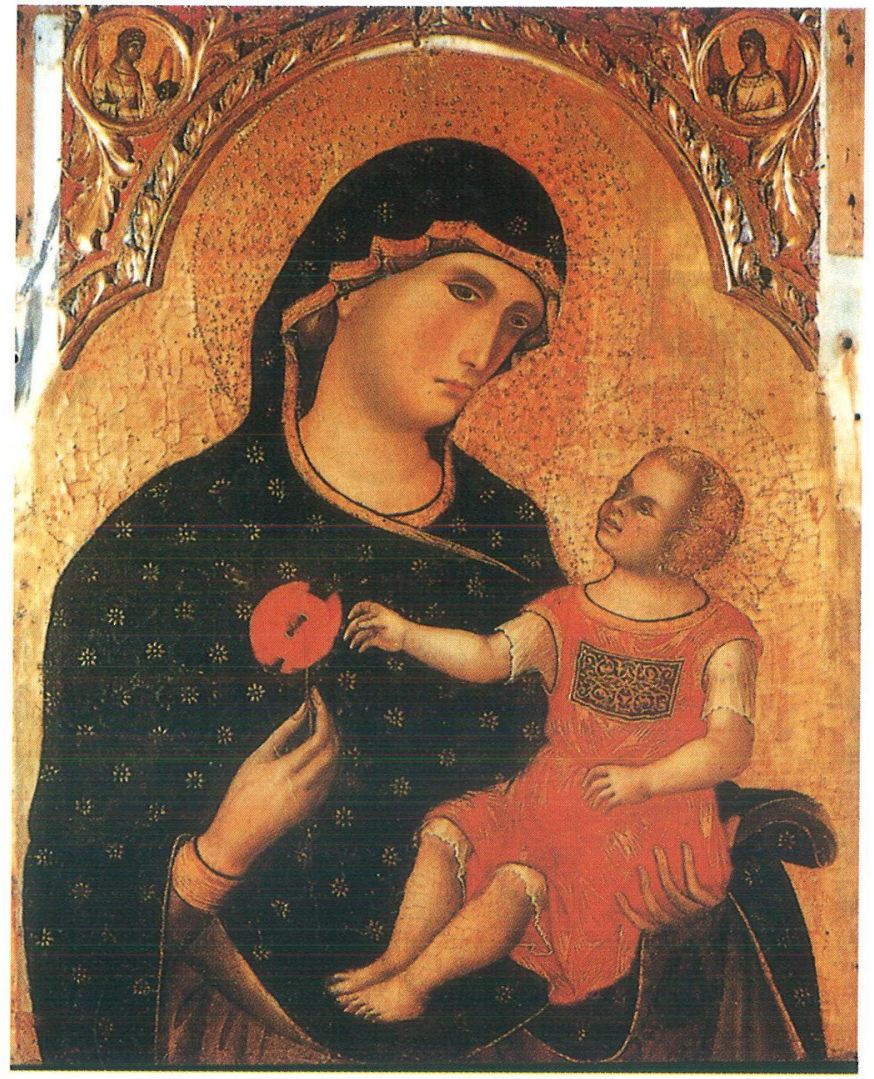

Fig. 4. Maestro Veneziano del primo Trecento, Madonna con Bambino; Venezia, Chiesa San Pantalon aggio gotico. E di questo goticismo anzi, nel contesto dell' arte padana, proprio Paolo è uno dei più alti interpreti, e la sua opera si connota addirittura di esiti che paiono preludere al gotico internazionale.

La sua prima opera conosciuta e sicuramente datata (1333) è il frammentario Polittico con la "Dormitio Virginis" proveniente dalla chiesa di San Lorenzo di Vicenza e ora al Museo Civico della città (fig. 5). La parte centrale del Polittico presenta una "Dormitio": essa è, all' inizio della attività a noi nota del Maestro, un esempio interessantissimo di come Paolo sappia tradurre in un nuovo modernissimo linguaggio gotico, del tutto aggiornato, $\mathrm{o}$ addirittura anticipatore rispetto alle coeve espressioni dei centri padani, la tradizione iconografica e tipologica bizantina. Si possono fare confronti e con la "Dormitio" mosaicata nella chiesa della Karie Cami a Costantinopoli, databile attorno al 1310, e con la tavoletta del Polittico di San Pantalon a Venezia, forse collocabile ancora entro il terzo decennio. Paolo rifiuta la rigida composizione simmetrica, scartando appena verso destra la mandorla con il Cristo rispetto alla cuspide della tavola, ciò che dà un leggero dinamismo alla composizione; la figura della Vergine distesa, si alleggerisce e diventa incorporea, avvolta in un morbidissimo manto che sembra fluire attorno al corpo, senza sottolinearlo, mentre l'orlo della stoffa è diventato un rivolo elegantissimo che fluisce con ritmo dolcissimo; infine alle ali lunghe e taglienti degli angeli è affidato di chiudere in maniera precisa la composizione attorno alla figura di Cristo. All'apparenza dunque la tradizione, ma sostanzialmente un radicale cambiamento linguistico, che è una traduzione in linguaggio ormai
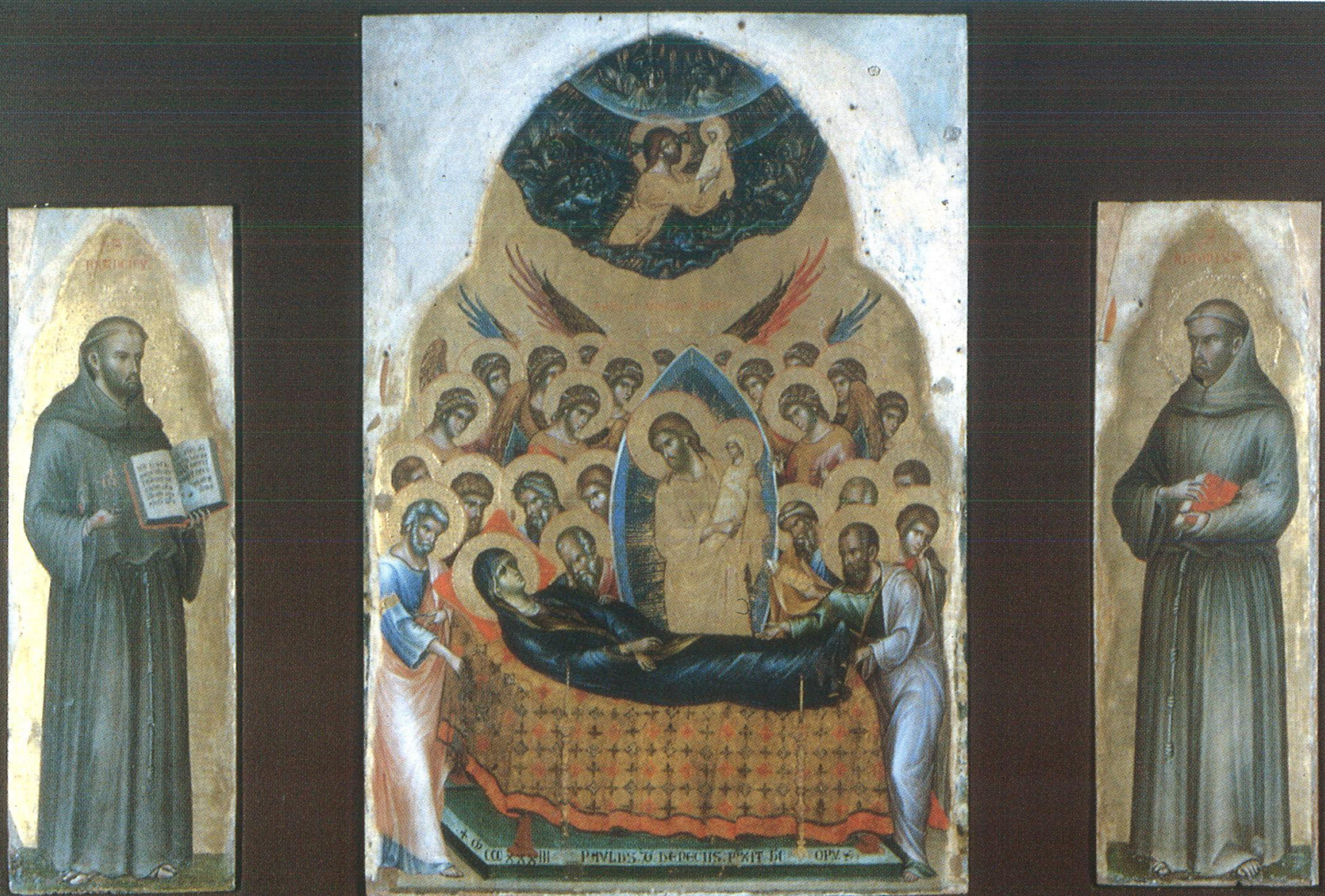


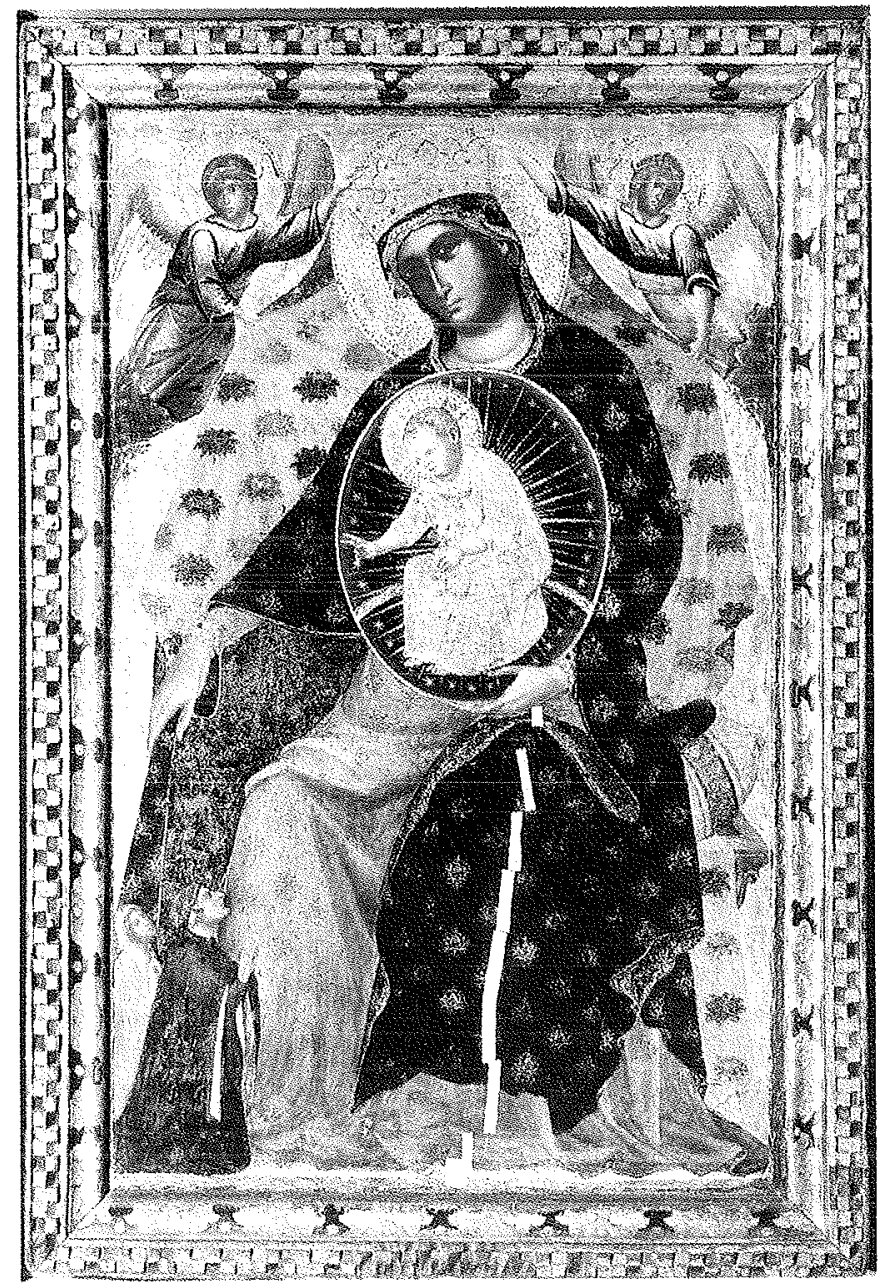

Fig. 6. Paolo Veneziano, Madonna e bimbo e gli offerenti; Venezia, Gallerie dell'Accademia

pienamente gotico. Vale la pena di pensare alla data della tavola vicentina, il 1333 , la stessa del Trittico con la "Crocifissione, l' Incoronazione e Santi", ora al Museo del Louvre, dal quale si fa iniziare la svolta moderna nella pittura bolognese, e si potrà osservare la maggiore e sostanziale modernità del dipinto paolesco rispetto a quello bolognese.

E il percorso figurativo di Paolo continua in questa direzione gotica, e dunque moderna e aggiornata: egli infatti partecipa del clima fortemente gotico, e già in certo senso "intemazionale", che caratterizzava in Venezia attorno agli anni centrali del Trecento la cultura figurativa più avanzata e moderna, e la cui massima espressione erano la architettura, totalmente innovativa, del Palazzo Ducale e le decorazioni fiorite dei suoi capitelli, opera straordinaria di Felice Calendario.

Le due tavolette con storie di San Nicola, già collezione Contini Bonacossi, oggi a Firenze a Palazzo Pitti, forse databili entro il quarto decennio, più che riferimenti all'arte giottesca, sono anch' esse una libera interpretazione di un modo narrativo e domestico, tipico del mondo veneziano già dai più antichi mosaici marciani. Ma vorrei anche sottolineare le somiglianze appunto con le figurazioni dei capitelli di Palazzo Ducale, per l' insistenza con cui sono resi i costumi, eleganti e alla moda, dei personaggi.

Una serie di tavole con la raffigurazione della Madonna col Bambino, scandite entro il quarto decennio del Trecento, nella sempre maggiore accentuazione di elementi gotici, indicano un percorso sempre più moderno e del tutto aggiornato con la pittura dei centri limitrofi.

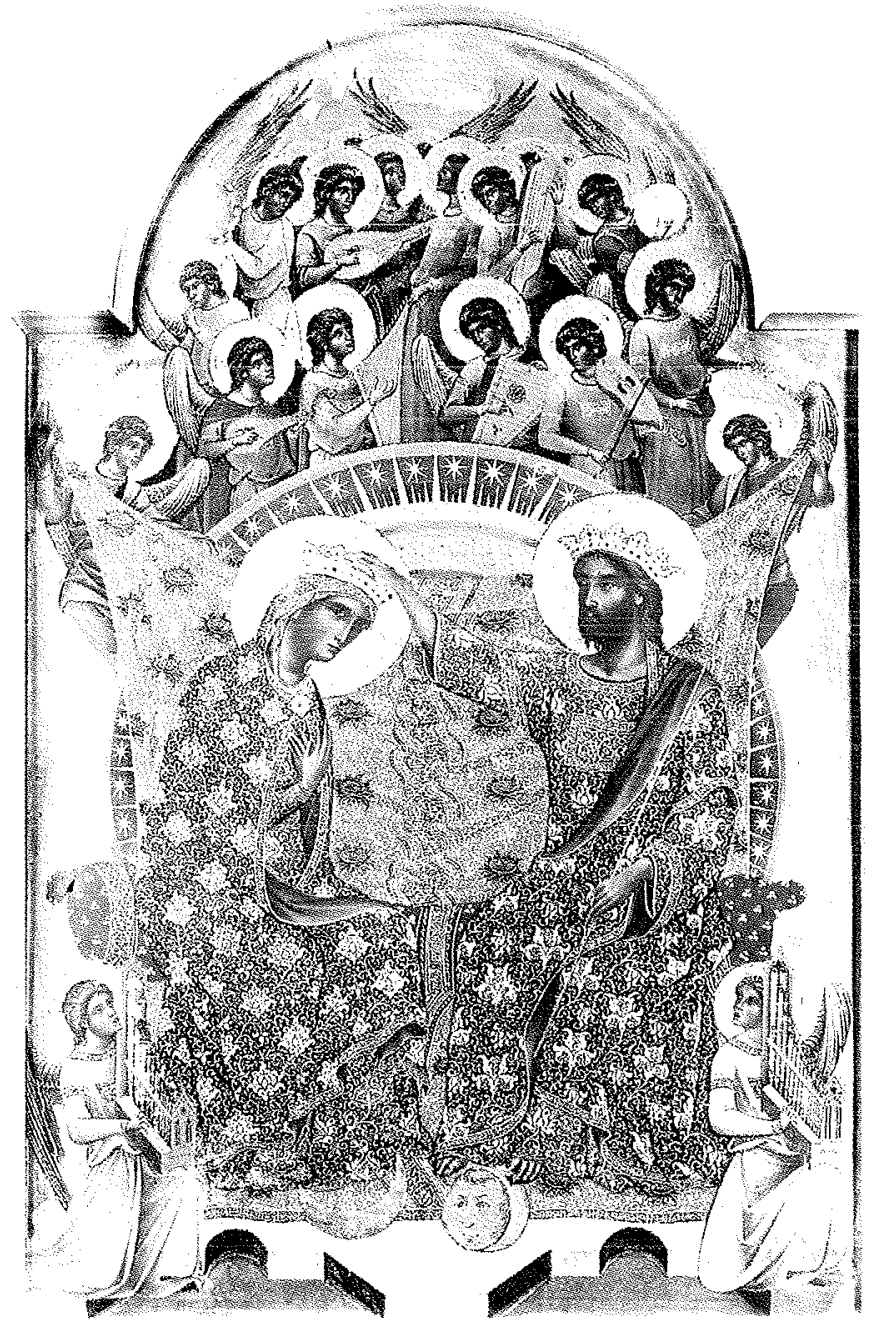

Fig. 9. Paolo Veneziano, "Incoronazione della Vergine", particolare del Polittico; Venezia, Gallerie dell'Academia

La lunetta della tomba di Francesco Dandolo ai Frari, databile attorno al 1339, con la Madonna e il Bambino, il Doge e la Dogaressa e i loro Santi protettori, è da leggersi come una traduzione gotica della immagine Edigitria: la composizione è calibrata in un gioco equilibratissimo e sottile di pieni e di vuoti, in un susseguirsi di linee delicatamente ricurve, che diventano tenerissime falcature nel manto della Vergine; il colore è superbamente acceso, nella luminosità dei gialli arancio e degli azzurri: gialli e azzurri costellati di grandi corolle di fiori, sulle stoffe preziose, tipiche della pittura veneziana.

Interessante è la grande "Madonna col Bambino" oggi all' Accademia di Venezia (fig. 6), all'apparenza bizantina, nel cupo pallore del volto, in realtà la icona della "Madre di Dio nel segno", è diventata una raffigurazione teneramente umana nella delicatezza del gesto, che accoglie sotto il manto gli offerenti, e inoltre una elegantissima regina nella ricchezza delle vesti e dei gioielli.

Seguono la Madonna di Sant' Alvise, che traduce in termini moderni una struttura orientale, quella della Casa del Clero a Padova, arricchita di preziose stoffe veneziane, e infine la Madonna della collezione Crespi, datata 1340 , il cui trono è suggerito da modelli continentali e gli angioletti sembrano echeggiare modelli continentali.

Sembra dunque che Paolo sia aperto a tutte le suggestioni, specie iconografiche, che gli vengono da altri centri pittorici, captandone immediatamente le novità tipologiche, ma trasformandole in un linguaggio personalissimo, fatto di ricordi orientali, e di splendori veneziani, in una resa tenerissima e 

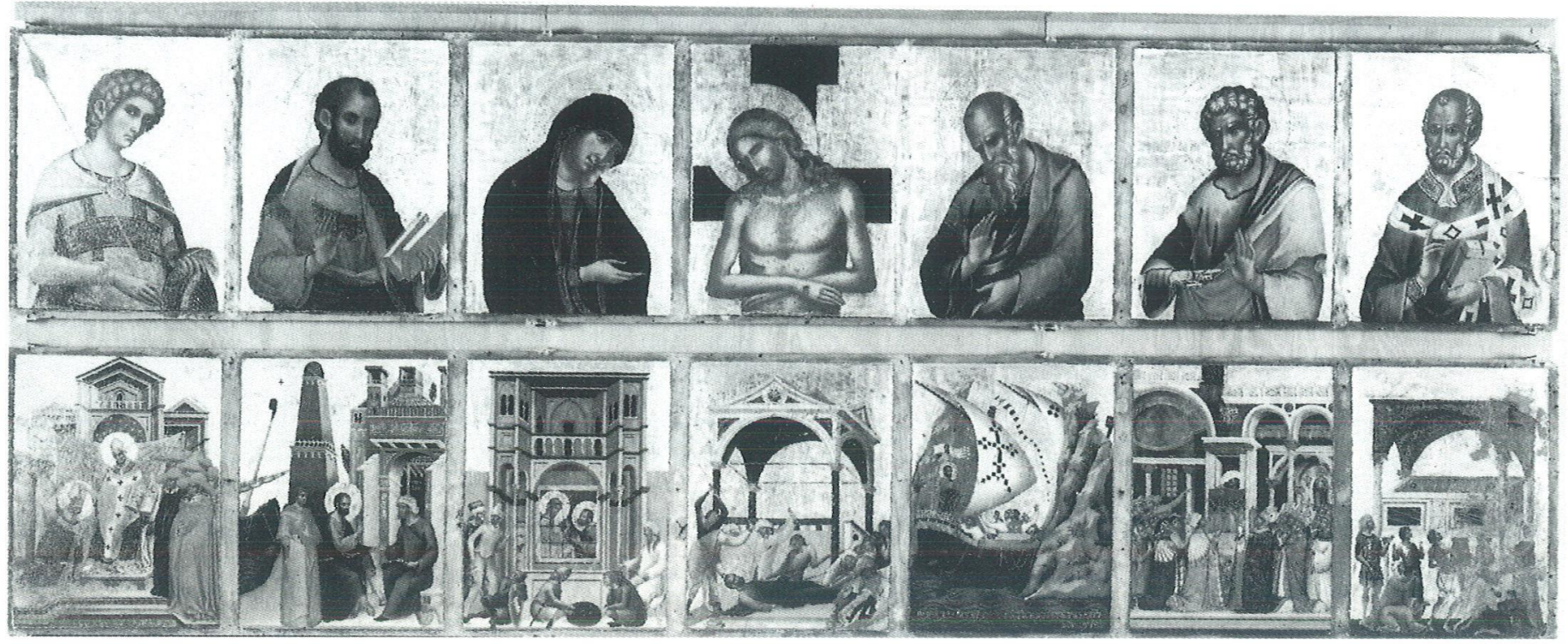

Fig. 7. Paolo Veneziano e figli Luca e Giovanni, Pala feriale; Venezia, Basilica di San Marco, Museo Marciano

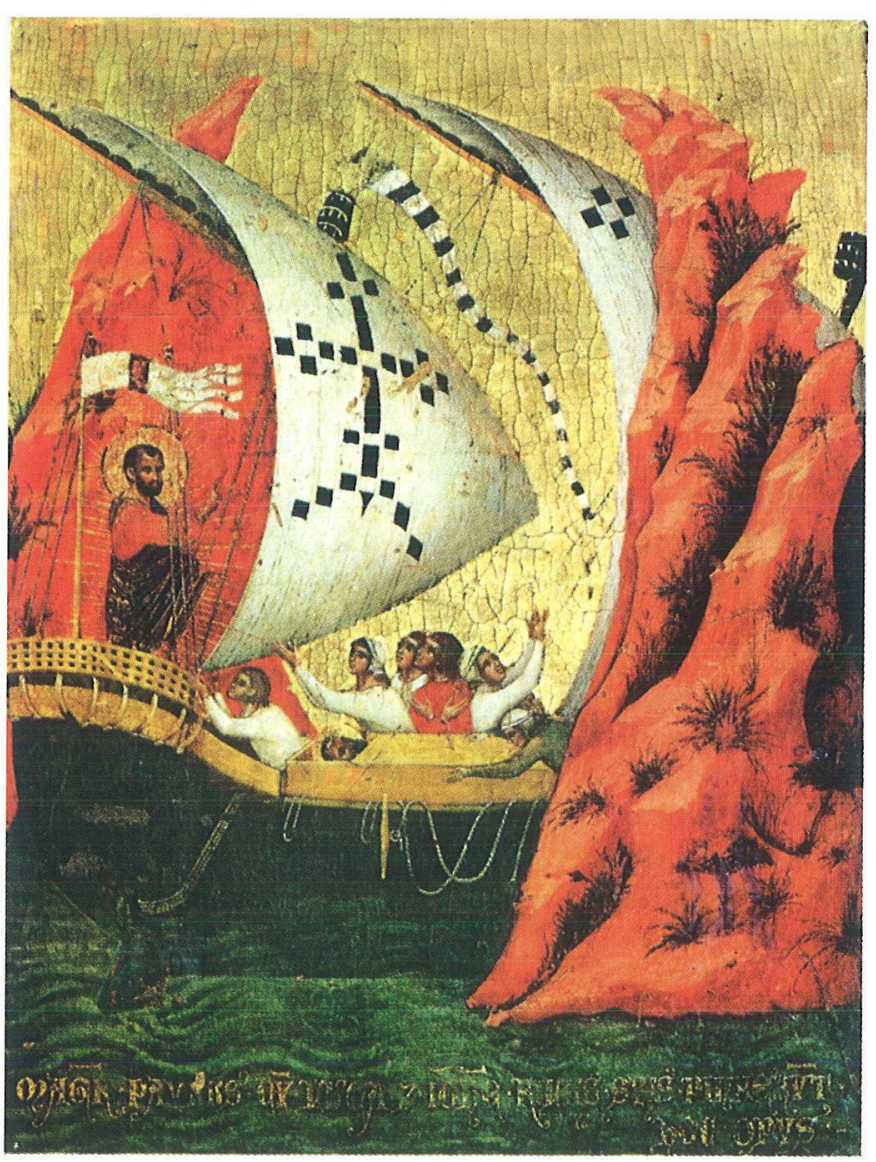

Fig. 8. Paolo Veneziano, Pala feriale (particolare)

delicatissima di linee e di colori, che costituiscono la peculiarità del suo stile, specie se rapportato a quello dei suoi numerosissimi seguaci e allievi.

Interessante è anche l'interpretazione che Paolo fa del mondo giottesco, che pure gli preme alle spalle, e che tuttavia gli è totalmente estraneo e per il senso dello spazio razionalmente misurato, e per il volume possente, costruito con il chiaroscuro. Traduzione gotica di modelli giotteschi, è ad esempio la piccola affascinante Crocifissione della Galleria Nazionale di
Washington ove il gruppo delle pie Donne, totalmente prive di corposità, come un flusso di linee ondeggiati, è un felicissimo tradimento del linguaggio giottesco; e gli angioletti azzurri attorno alla Croce sembrano preludere a Stefan Lochner. La patetica dolcissima Croce di San Samuele, ora nella Sacrestia di Santo Stefano, esemplata nella tipologia del corpo, e nella struttura delle gambe e delle mani, sui modelli giotteschi - la Croce del Museo di Padova in particolare - sia pure attraverso il filtro di alcuni stilemi tradizionali, quali la testa piccola e la estenuata esilità delle membra, è ancora una volta una interpretazione in chiave gotica del modello, come emerge nella raffinatezza del perizoma trasparente e nella duttile delicatezza del segno di contorno.

Particolarmente interessante per misuare la capacità inventiva di Paolo, ma allo stesso tempo la radicalità di un legame con la tradizione bizantina, è la pala feriale marciana del 1345 (figg. 7-8). Le sette storiette di San Marco nel registro inferiore del dipinto sono tutte in chiave moderna, e amplificano il discorso immediato e narrativo proprio del linguaggio lagunare, con alcune punte innovative significative, come le architetture di sfondo della basilica, classicheggianti, e i gruppi dei prelati, che paiono anticipare addirittura i più antichi dipinti di Antonio Vivarini.

Le sette tavole della fascia superiore sembrano invece tradizionali: e lo sono, ma solo nella scelta dei soggetti: mezze figure di Santi, di fattura allungata e dai volti chiusi e bruniti. Di fatto la morbidezza del segno di contorno, scorpora quelle figure in ritmi assolutamente gotici - si vedano le mani, o l'orlo del mantello - e i colori si accendono di contrasti vivissimi, estranei alla tradizione bizantina.

Il percorso posteriore della pittura di Paolo va sempre più nella direzione addirittura di anticipazioni del gotico internazionale. Così ad esempio nella "Madonna col Bambino" di Carpineta (1347), rivestita di un modernissimo tessuto tutto ricamato, nella elegantissima "Incoronazione della Vergine" sul Polittico oggi all'Accademia di Venezia (fig. 9), o nella figura bellissima della Sant' Orsola del polittico del Museo Civico di San Severino Marche, felicissimo preludio, nello splendore delle vesti cortesi, al gotico internazionale.

Il percorso a noi noto di Paolo termina con l'"Incoronazione della Vergine" della collezione Frick, eseguita con l'aiuto del figlio Giovanni, datata 1358, totalmente gotica, nell'al- 


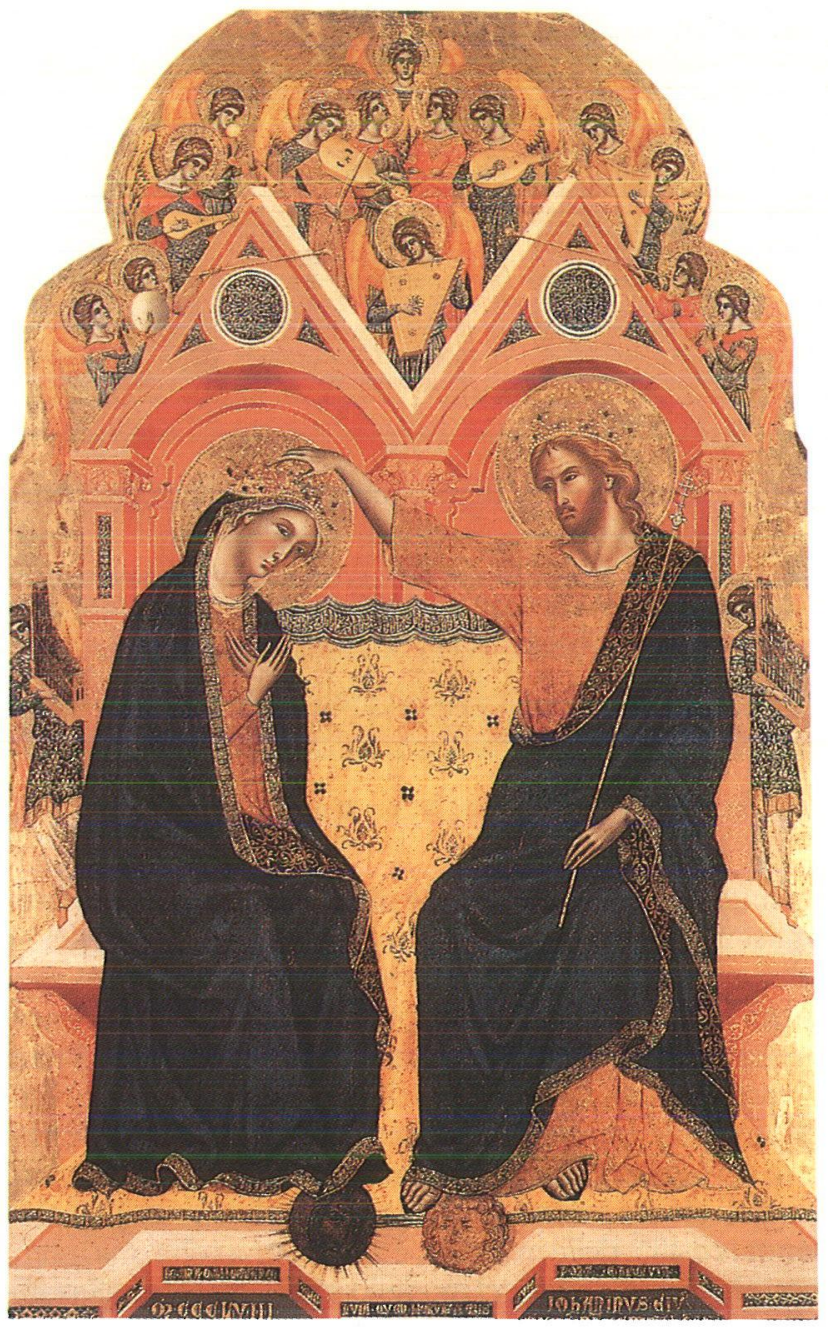

Fig. 10. Paolo Veneziano e Giovanni, "Incoronazione della Vergine"; New York, Collezione Frick

lungarsi senza spessore delle due figure e nello splendore degli accostamenti di colore (fig. 10).

E tuttavia la sottile e allo stesso tempo radicale rivoluzione del linguaggio di Paolo avrà esiti risolutivi solo nell' altro grande pittore veneziano, Lorenzo. Attorno a Paolo solo pochi artisti sembrano voler cogliere alcune novità del suo linguaggio, come il pittore del Dossale con Storie di Santa Lucia del Vescovado di Krk, attribuito del resto anche a Paolo, o il pittore del paliotto con le Storie di Sant' Orsola, di collezione privata fiorentina: ambedue opere cariche di briosa fantasia narrativa.

I numerosi esempi di dipinti del primo Trecento mostrano ancora stretti legami con la tradizione, senza che il linguaggio gotico porti alcun mutamento. Si potranno osservare a questo proposito il "Crocifisso" di San Beneto, quello ancor più tra-

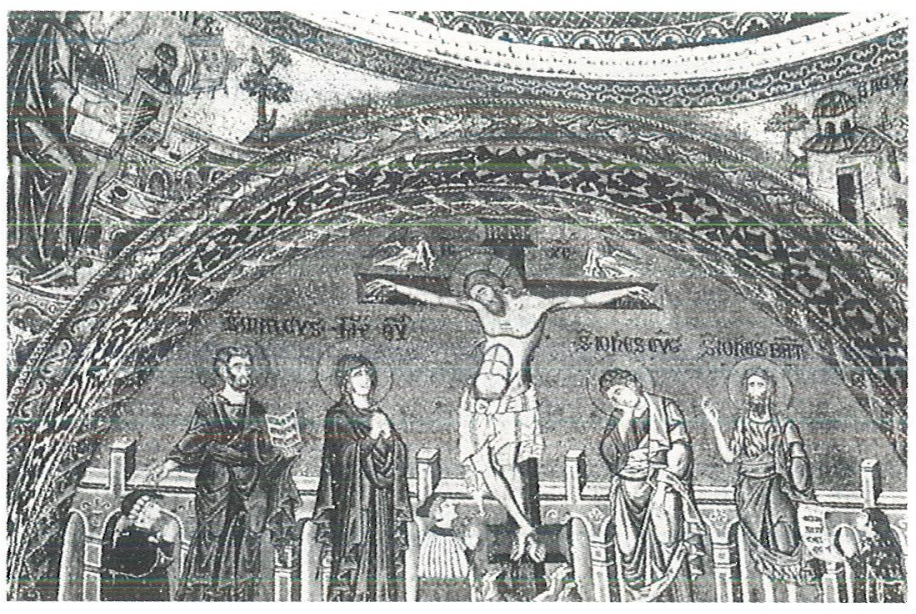

Fig. 11. Mosaicista veneziano attivo alla metà del XIV secolo, Crocifissione; Venezia San Marco, Battistero

dizionale del Museo Correr, e infine quello dell' Istituto Ellenico di Venezia, per verificare quanto poco i pittori contemporanei a Paolo abbiano compreso la sua conversione moderna.

Ancor più sorprendente è la tavola con la "Crocifissione tra Santi” del Museo Correr, che esibisce ancora una ambientazione del tutto tradizionale, e il frammento di "Crocifissione" a fresco, recentemente recuperato in un ambiente adiacente la chiesa di San Nicolò dei Mendicoli. ${ }^{14}$

Piuttosto sconcerta di più il fatto che anche nelle commissioni ufficiali si prediliga il linguaggio tradizionale: il caso più clamoroso è quello della decorazione del Battistero marciano (fig. 11), voluto da Andrea Dandolo, tra il 1345 e il 1354. Sia dal punto di vista iconografico, che da quello della resa dei singoli personaggi (fig. 12), ci troviamo di fronte ad un' opera assolutamente legata alla tradizione. Solo i due episodi della Salomé (fig 13) sono trattati in chiave moderna, la stessa che ci dà, e forse si tratta degli stessi mosaicisti, la decorazione, assolutamente originale, della cappella di Sant'Isidoro, databile attorno al 1355 . Sembrerebbe dunque che il discrimine tra tradizione bizantina e moderno mondo gotico dovesse situarsi attorno al 1350.

Ma non è così: esistono come si è visto, opere, sia pure di datazione tuttora incerta, che possono situarsi negli anni a cavallo della metà del secolo che si propongono in chiave assolutamente "bizantina". E il filone tradizionale non è, anche più tardi, assolutamente perdente. Nel 1372 infatti Donato e Catarino firmano e datano una preziosa "Incoronazione della Vergine", ora alla Querini Stampalia, ancora in chiave tradizionale, anche se arricchita di preziosissimi ori e gemme.

Ẽuna prova che la tradizione in Venezia continua e si intreccia con i filoni più moderni e aggiornati della pittura veneziana, ma costituendo una costante, si può dire per tutto il secolo e oltre.

\footnotetext{
' Per il problema dell'arte veneziana e i suoi legami con quella bizantina cito qui solamente: G. FIOCCO, Bisanzio, Ravenna, Venezia, in "Rivista di Venezia", 2, 1930; P. TOESCA, Il Trecento, Torino 1951, pp. 688-718; R. PALLUCCHINI, La Pittura veneziana del Trecento, Venezia-Roma 1964, passim; P. TOESCA, Il Medioevo II, Torino 1965, pp. 982-985; V. LAZAREFF, Storia della pittura bizantina, Torino 1967, pp. 406-408. O. DEMUS, Bizantine Art and the West, London 1970, passim; S. BETTINI (a cura di), Venezia e Bisanzio, Catalogo della Mostra, Venezia 1974.

${ }^{2}$ O. DEMUS, The Mosaics of San Marco in Venice, Washington 1984, passim, e O. DEMUS, The Mosaic Decoration of San Marco Venice, Chicago 1988, passim.
} 


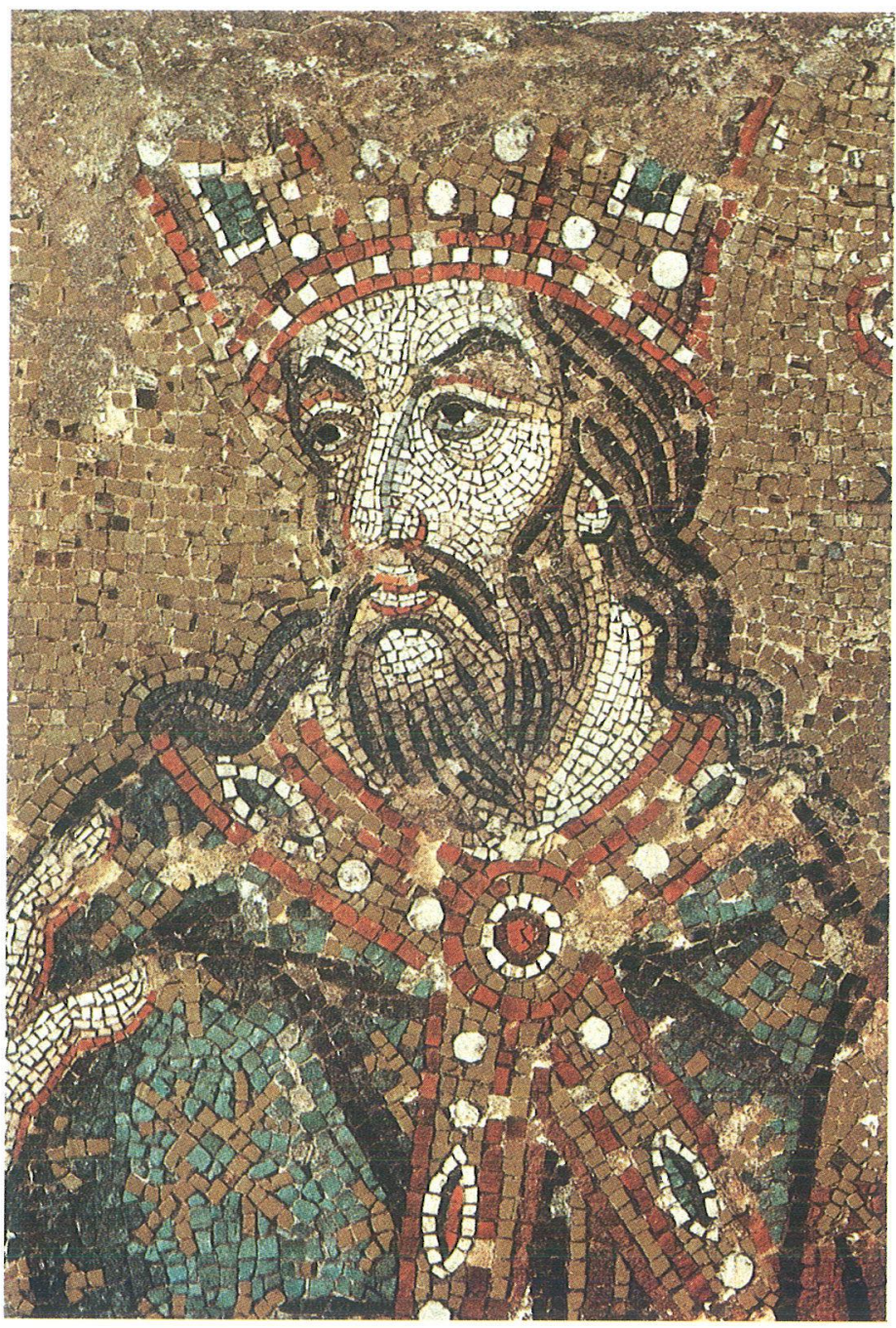

Fig. 12. Mosaicista veneziano attivo alla metà del XIV secolo, Particolare della "Adorazione dei Magi"; Venezia, Museo Marciano

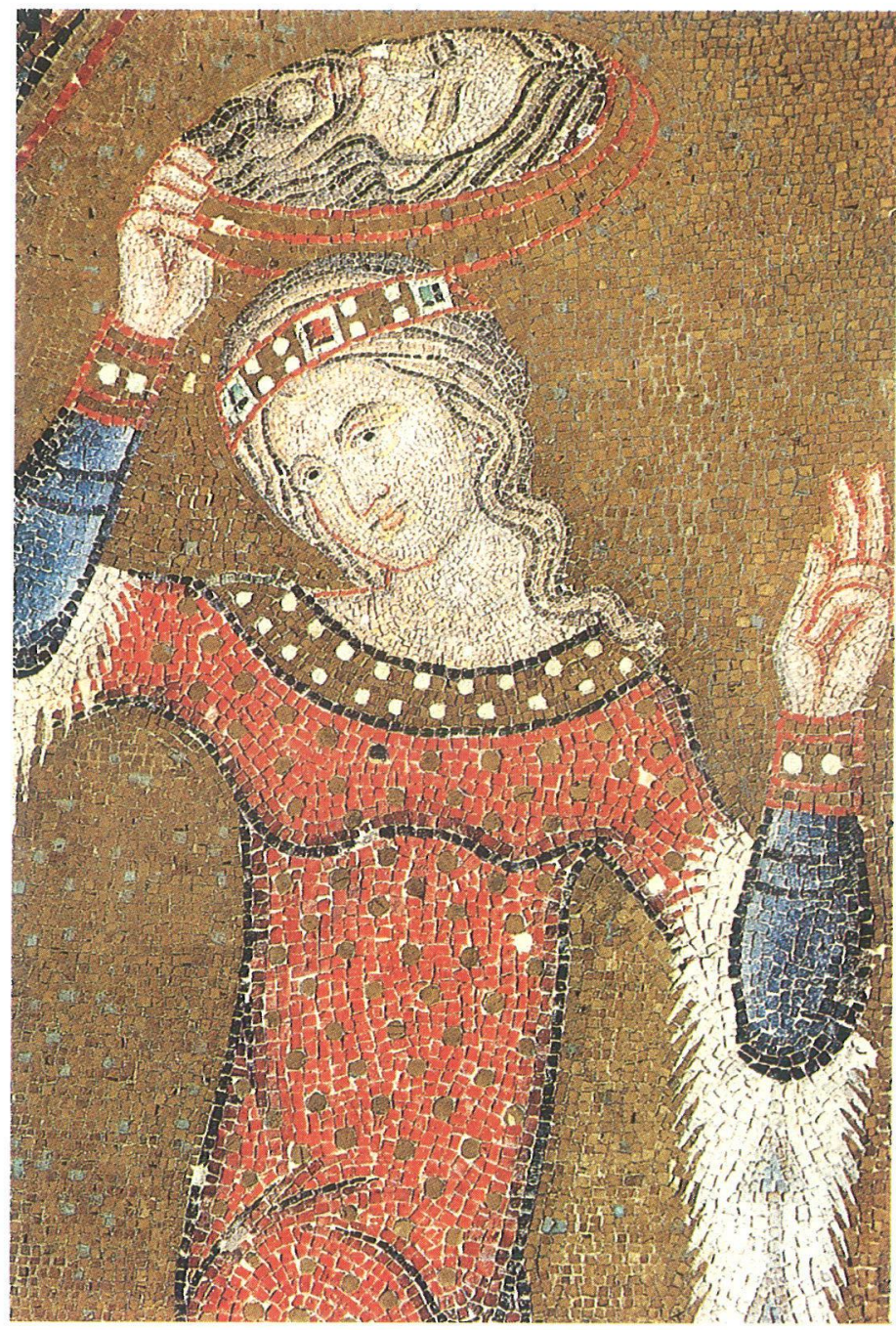

Fig. 13. Mosaicista veneziano della metà del XIV secolo, Salomé (part.); Venezia, San Marco, Battistero

${ }^{3}$ G. FIOCCO, Gli affreschi bizantini di San Zan Degolà, in "Arte Veneta", 1951; M. MURARO, Antichi affreschi veneziani, in "Le Meraviglie del passato", Milano 1974; M. MURARO, Scheda relativa, in Venezia e Bisanzio-Catalogo della Mostra, Venezia (ma Milano) 1974, s.p.; A. PAGNES, Uno studio per gli affreschi medievali di San Zan Degolà, Venezia 1987; F. d' ARCAIS, Venezia, in AA. VV. La pittura nel Veneto, Il Trecento, I, Milano 1992, p.19.

${ }^{4}$ Operette attribuite a Paolo o ad ambito paolesco: si veda R. PALLUCCHINI, op. cit., pp. 20-21, e la scheda relativa al dipinto in M. MURARO, Paolo da Venezia, Milano 1969 p.132, (con ampia bibliografia) dove le tavolette sono assegnate a Maestro Veneto attorno al 1350.

${ }^{5}$ Il Paliotto fu fatto conoscere da A. MORASSI (Il fondo oro trecentesco e la predella belliniana di Dignano, in "Rivista della città di Venezia", 1923, 9) e attribuito a Paolo Veneziano da G. FIOCCO (Le primizie di Maestro Paolo Veneziano, in "Dedalo", IV, 1930-31): l'attribuzione è tuttavia controversa. Il dipinto fu presentato alla Mostra tenuta a Zagabria nel 1967 come opera di "predecessore" di Paolo Veneziano (V. ZLAMALIK, Paolo Veneziano $i$ njegov krug - Catalogo della Mostra, Zagreb 1967, pp. 12-19, con bibliografia precedente). Non accetta l' attribuzione a Paolo M. MURARO, ( Paolo da Venezia, op. cit., pp. 25-27), che ritiene il paliotto opera di un artista veneziano attorno al 1350.

${ }^{6}$ E. B. GARRISON, Italian Romanesque Panel Painting, Firenze 1949.

${ }^{7}$ Si veda in particolare V. LAZAREFF, Über eine Gruppe byzantinisch-venezianischer Trecento-Bilder, in "Art Studies" 1931; IDEM, Saggi sulla pittura veneziana de secoli XIII-XIV. La maniera greca e il problema della maniera cretese, in "Arte Veneta" I, 1965, pp. 23-25.

${ }^{8}$ V. LAZAREFF, Saggi...cit., pp. 23-25; accettano questa ricostruzione, tra gli altri il MURARO (Paolo da Venezia, op. cit., pp. 157-158); F. R. SHAPLEY, Catalogue of the Italian Paintings, vol. I, Washington National Gallery of Art, 1976, pp. 326-328, con completa bibliografia precedente, F. ZULIANI (vedi nota 10), e F. d' ARCAIS, Venezia, op. cit., p. 23;

${ }^{9}$ V. LAZAREFF, Über eine Gruppe.., op. cit.; e IDEM, Saggi.., op. cit., pp. 26-28.

${ }^{10}$ Per le opinioni relative alla tavoletta del Museo Civico di Padova si veda la scheda, con ampia bibliografia precedente, di F. ZULIANI, in Da Giotto al Tardogotico - Catalogo della Mostra, Museo Civico di Padova, Roma 1989, pp.77-79, dove l' opera reca l' attribuzione al "Maestro della Incoronazione della Vergine".

${ }^{11}$ Di attribuzione controversa: vedi R. PALLUCCHINI, La Pittura, op. cit., p. 25; M. MURARO (Paolo da Venezia, op. cit., p. 146), propone una attribuzione ad un seguace di Paolo, attivo verso il 1350. Una datazione tarda è riproposta (saggio in corso di stampa) dal dott. Sandro Sponza.

${ }^{12}$ Su questo importante dipinto lo studio più completo è quello di M. WALCHER CASETTI, Il Trittico di Santa Chiara di Trieste e l' orientamente paleologo nell'arte di Paolo Veneziano, Trieste 1961. Per V. LAZAREFF (Saggi.., op. cit., p. 21 e n. 22) il dipinto fa parte di un folto gruppo di opere, di pittori veneziani, che si caratterizzano per un fare miniaturistico. 
${ }^{13}$ Per Paolo Veneziano rimando ad alcune opere fondamentali, a loro volta ricche di una bibliografia completa sull' argomento: illuminanti sono le poche righe che P. TOESCA (Il Trecento, op, cit. pp. 706-708) dedica al pittore; si veda inoltre: R. PALLUCCHINI, La Pittura veneziana del Trecento, Venezia Roma 1964, pp. 16-63; V. ZLAMALIK, Paolo Veneziano i njegov krug - Catalogo della Mostra, Zagreb 1967; M. MURARO, Paolo da Venezia, Milano 1969; vedi inoltre, più recentemente F. d' ARCAIS, Venezia, cit., pp. 24-42. con ampia bibliografia.

${ }^{14}$ Restauri a Venezia 1967-1986, "Quademi della Soprintendenza ai beni artistici e storici di Venezia", no. 14, Venezia 1986, p. 86.

\section{TRADICIJA I INOVACIJA U VENECIJANSKOJ SKULPTURI TRECENTA: PAOLO I OKO PAOLA}

\section{SAŽETAK}

Venecija je na polju slikarstva osebujna "bizantska" provincija od samog početka (mozaici iz Torcella i Svetoga Marka), pa najmanje do polovice XIV. stoljeća.

Djela iz prvih desetljeća XIV. stoljeća pokazuju izravan kontinuitet s onima iz prethodnog stoljeća, iskazujući snažnu povezanost s bizantskom umjetnošcu, kako carigradskom tako i onom iz provincija, pogotovo srpskom i makedonskom umjetnošću.

Ta tijesna povezanost prekida se s Paolom Venezianom; Majstorova djela, iako $u$ nekim aspektima oslonjena na tradiciju, izgledaju sve više gotički, moderno, osuvremenjena na slikarstvu padovanskog područja, a također u vezi s prekoalpskom gotikom. Paolov umjetnički put usmjerava se sve više prema elegantnom, rafiniranom, dragocjenom izrazu koji u kasnijim djelima kao da nagovješćuje "internacionalni stil" kasne gotike, paralelno s najnovijim izrazima arhitekture i skulpture (Duždeva palača i pripadajući kapiteli Filippa Calendarija). Međutim, Paolovi sljedbenici ne razumiju učiteljeve inovacije i zato se može reći da gotički izraz u Veneciji predstavlja "gubitnički" pravac čiji je najistaknutiji predstavnik u drugoj polovici XIV. stoljeća Lorenzo Veneziano. Izrazi vezani za bizantski ukus nastavljaju se čitavom prvom polovicom stoljeća i poslije, može se reći sve do Donata i Catarina (Krunjenje Djevice iz 1372., Querini Stampalia).Začudo se i u službenim narudžbama daje prednost tradicionalnom izrazu barem do polovice stoljeća, kako pokazuju izrazito bizantski mozaici Krstionice Sv. Marka, izrađeni po narudžbi Dužda Andrea Dandola (1344-1354) i koji stoga mogu biti datirani unutar 1354. godine. 